\title{
Fatores de risco do déficit de estatura em crianças pré-escolares: estudo caso-controle
}

\author{
Risk factors of stunting in preschool children: a case-control study
}

Dixis Figueroa Pedraza ${ }^{1}$

Tarciana Nobre de Menezes ${ }^{1}$

${ }^{1}$ Programa de PósGraduação em Saúde Pública, Universidade Estadual da Paraíba. Av. das Baraúnas 351/Campus Universitário, Bodocongó 58.109-753 Campina Grande Paraíba Brasil. dixisfigueroa@gmail.com

Abstract This article seeks to establish risk factors for mild and moderate stunting among preschool children attending public daycare centers. It involves a case-control study with 67 pairs of children. Cases defined as children with height/ age between -1 and -2 Z-scores were paired with controls between -1 Z-score and the median, while cases with height/age $<-2 Z$-scores were paired with controls with height/age > median to $+1 Z$-score. The sex and age of the child was used for pairing purposes. The final model of hierarchical multivariate analysis indicated a greater chance of stunting in the following groups of preschoolers: family income $<1 / 2$ Minimum Wage, squatted/courtesy house, number of individuals in the household $\geq 6$, maternal age at child's birth $<20$ years, low maternal stature, incomplete immunization program, low birth weight. Stunting is determined by adverse socioeconomic conditions and the characteristics of children reflecting health care (maternal height and child variables).

Key words Height, Height/age, Growth, Preschool
Resumo O objetivo deste artigo é identificar fatores associados ao retardo leve e moderado de crescimento entre pré-escolares assistidos em creches públicas. Estudo caso-controle com 67 pares de crianças. Os casos definidos como crianças com estatura/idade entre - 1 e - 2 escore- $Z$ foram pareados a controles situados entre -1 escore- $Z$ e a mediana, enquanto casos com estatura/idade <2 escore- $Z$ foram pareados a controles com estatura/idade $>$ mediana até +1 escore- $Z$. O pareamento considerou o sexo e a idade da criança. $O$ modelo final da análise multivariada hierarquizada indicou maior chance de déficit estatural nos seguintes grupos de pré-escolares: renda familiar per capita < 1/2 SM, casa invadida/cedida, número de indivíduos no domicílio $\geq 6$, idade materna ao nascimento da criança $<20$ anos, estatura materna baixa, esquema vacinal incompleto, baixo peso ao nascer. A determinação do déficit de estatura indica importante influência das condições socioeconômicas adversas e de características que refletem cuidados de saúde (estatura materna e variáveis infantis).

Palavras-chave Estatura, Estatura-idade, Crescimento, Pré-escolar 


\section{Introdução}

Apesar das reduções significativas nos países em desenvolvimento, o déficit de estatura ainda continua sendo o principal problema de saúde pública em muitos deles ${ }^{1}$. Estimativas da Organização das Nações Unidas indicam que 34\% das crianças de todo o mundo apresentam déficit de estatura, percentual que aumenta para $44 \%$ quando analisados os países menos desenvolvidos ${ }^{2}$. No caso brasileiro, o problema apresentou marcantes reduções nas últimas décadas ${ }^{3}$, indicando-se como fatores importantes as melhoras relacionadas ao poder aquisitivo dos mais pobres e os avanços nos serviços essenciais de saúde, educação e saneamento ${ }^{3,4}$. Entretanto, essa melhora caracteriza-se por maiores prevalências que persistem entre as crianças de famílias menos favorecidas ${ }^{4}$, o que condiz com os conhecimentos sobre as iniquidades socioeconômicas em termos de retardo do crescimento linear ${ }^{5}$.

O comprometimento do crescimento linear contribui com as altas taxas de morbimortalidade e pode influenciar o desenvolvimento motor, mental e o desempenho escolar ${ }^{6,7}$. Esses efeitos negativos não são restritos aos casos mais severos de déficit estatural. As formas leves e moderadas de desnutrição crônica também aumentam o risco de mortalidade e contribuem com 50-75\% dos casos de doença nos primeiros anos de vida, além de representarem a possibilidade de modificar para casos de desnutrição mais severa associada ao acometimento de doenças ${ }^{8}$. Assim, a relevância epidemiológica do déficit de estatura compreende também as formas menos severas, nas quais os estudos de tendência e dos fatores responsáveis pela evolução têm centrado menos atenção.

Diante do exposto, o presente estudo teve por objetivo identificar fatores associados ao retardo leve e moderado de crescimento entre préescolares assistidos em creches públicas.

\section{Métodos}

Este estudo foi planejado sob a forma de um desenho tipo caso-controle com a finalidade de verificar fatores associados ao déficit de estatura em crianças pré-escolares assistidas em creches públicas do município de João Pessoa pertencentes à Secretaria de Estado do Desenvolvimento Humano da Paraíba. Essa Secretaria gerenciava um total de 45 creches na época da pesquisa, das quais 30 estavam situadas no município de João
Pessoa, capital do estado, beneficiando um total de 2800 crianças.

Os grupos de casos e controles do presente estudo foram obtidos de um levantamento prévio realizado em 11 creches incluindo 340 crianças selecionadas aleatoriamente. O levantamento incluiu somente uma criança de cada família. Foram formados dois grupos de casos (grupo caso 1: Ca1, grupo caso 2: Ca2) e dois grupos de controles (grupo controle 1: Col, grupo controle 2: Co2). A formação dos grupos foi condicionada ao escore- $Z$ da estatura/idade em relação à mediana da população de referência: Cal, crianças com estatura/idade < -2 escore-Z; Ca2, crianças com estatura/idade entre - 1 e - -2 escore-Z; Col, crianças com estatura/idade $>$ mediana da população de referência até +1 escore-Z; Co2, crianças com estatura/idade entre -1 escore- $Z$ e a mediana da população de referência. $O$ emparelhamento das crianças obedeceu a um ordenamento sequencial decrescente dos valores de escore- $\mathrm{Z}$ apresentados pelos casos. Crianças do grupo Cal foram pareadas a crianças do grupo Co1, do mesmo sexo e idade, e crianças do grupo $\mathrm{Ca} 2$ foram pareadas a crianças do grupo $\mathrm{Co} 2$, do mesmo sexo e idade. O pareamento para a idade considerou a variabilidade de um mês para mais ou para menos.

As medidas de estatura foram coletadas por uma equipe de antropometristas treinados e padronizados obedecendo aos procedimentos recomendados pela Organização Mundial da Saú$\mathrm{de}^{9}$. As crianças menores de dois anos tiveram o comprimento medido por meio de antropômetro infantil de madeira (Alturexata ${ }^{\circledR}$ ) com amplitude de $130 \mathrm{~cm}$ e subdivisões de $0,1 \mathrm{~cm}$. As crianças de dois anos ou mais tiveram a altura medida por meio de estadiômetro (WCS ${ }^{\circledR}$ ) com amplitude de $200 \mathrm{~cm}$ e subdivisões de $0,1 \mathrm{~cm}$. As medições foram realizadas em duplicata e a medida final resultou da estimativa da média das duas medições. O índice antropométrico estatura/idade foi calculado para cada criança segundo os valores em escore-Z. Os cálculos foram realizados com os programas WHO Anthro 2009, para crianças abaixo de cinco anos de idade, e WHO Anthro Plus 2009, para crianças com cinco anos ou mais. Como população de referência, adotou-se a do Multicentre Growth Reference Study, atualmente recomendado pela Organização Mundial da Saúde ${ }^{10,11}$.

O tamanho da amostra, 72 pares (72 crianças com déficit de estatura e igual número de crianças controle), foi calculado para um nível de confiança de $95 \%$, um poder do teste de $80 \%$, 
$30 \%$ de fatores negativos nas crianças eutróficas e uma razão de chances (OR) de 1,8 nas crianças com déficit de estatura. Das 340 crianças sorteadas para o estudo, 89 não se enquadraram em nenhum dos grupos predeterminados. Das 251 crianças que restaram, 19 foram enquadradas em Ca1, 76 em Ca2, 48 em Co1 e 108 em Co2. Ao parear, formaram-se 10 pares de crianças do mesmo sexo e idade de Ca1 com Co1 e 57 de Ca2 com Co2, totalizando 67 pares do total necessário (72).

Para obtenção das informações necessárias à caracterização de fatores de risco do déficit de estatura, foram realizadas entrevistas com a mãe ou o responsável pela criança. As entrevistas foram realizadas nas próprias creches que as crianças frequentavam por pessoal treinado com o instrumento de coleta de dados. O questionário continha informações sobre condições socioeconômicas da família (obtidas da ficha da criança constante na creche), antecedentes maternos e características das crianças. As variáveis independentes de estudo foram:

- condições socioeconômicas: renda familiar per capita, tipo de casa, número de cômodos no domicílio, número de indivíduos no domicílio;

- antecedentes maternos: suplementação com sulfato ferroso na gravidez, número de consultas de pré-natal, uso de cigarro na gravidez, idade ao nascimento da criança, estatura para idade (a baixa estatura materna foi definida pelo ponto de corte $155,0 \mathrm{~cm}^{12}$ );

- características das crianças: sexo, esquema vacinal (obtido da Caderneta de Saúde da Criança), peso ao nascer (obtido da Caderneta de Saúde da Criança).

Para avaliar a associação das variáveis independentes com o crescimento linear das crianças, atendeu-se às especificações dos modelos hierarquizados, utilizando três níveis de determinação: distal (condições socioeconômicas), intermediário (antecedentes maternos), proximal (características das crianças $)^{13}$. Foram elaborados modelos de regressão logística segundo técnica condicional, passo a passo, com as variáveis que apresentaram associação significativa dentro de um limite de aceitabilidade, p de até 10\%, nas análises bivariadas, pela técnica da MantellHaenszel. O ingresso no modelo de regressão obedeceu, além da significância, à hierarquia do modelo teórico adotado. Testes de correlação múltipla foram realizados para verificar viés decorrente de multicolinearidade, mantendo-se apenas um dos fatores de risco, sempre que houvesse correlação entre os mesmos $(r=0,5$ e $p<$
0,01). Foi considerado o nível de significância de $5 \%$ para indicar associação. Os softwares utilizados foram o Excel 2000 e o Stata v. 7.0.

Todas as diretrizes éticas da Resolução no 196/ $96^{14}$ do Conselho Nacional de Saúde, bem como os princípios éticos referentes às publicações de pesquisa com seres humanos contidos na Declaração de Helsinki foram contempladas e o projeto foi aprovado pelo Comitê de Ética em Pesquisa da Universidade Estadual da Paraíba.

\section{Resultados}

Dentre os 134 pré-escolares pertencentes à amostra, 53,0\% eram do sexo masculino e $47,0 \%$ do feminino. Verificou-se que $68,6 \%$ das crianças estavam com o esquema vacinal completo e que $83,6 \%$ nasceram com peso adequado, com menor quantidade de crianças do grupo de caso nessas situações. Com relação aos antecedentes maternos, observou-se que, entre os pré-escolares com desnutrição por déficit estatural, em $34,3 \%$ dos casos as mães fumaram durante a gravidez, em relação a 29,8\% do grupo controle; em $29,8 \%$ as mães tinham menos de 20 anos ao momento do nascimento, em relação a 16,4\% do grupo controle; e em $35,8 \%$ as mães eram de baixa estatura, em relação a $19,4 \%$ do grupo controle. Para as características socioeconômicas, 94 famílias possuíam renda familiar per capita inferior a meio salário mínimo, sendo 54 entre o grupo de caso; e 101 residiam em casa invadida/cedida, sendo 55 entre o grupo de caso.

A Tabela 1 apresenta os resultados referentes às análises de associação da baixa estatura com as variáveis referentes às condições socioeconômicas das famílias, antecedentes maternos e características das crianças. Mostraram-se associadas à presença de estatura baixa, a renda familiar, o tipo de casa, o número de indivíduos no domicílio, o uso de cigarro na gravidez, a idade da mãe ao nascimento da criança, a estatura para idade da mãe, o esquema vacinal da criança e o peso ao nascer. Pré-escolares cujas rendas familiares per capita foram menores que 1/2 salário mínimo (SM) apresentaram chance 2,8 vezes maior de estatura baixa que aqueles de famílias com renda igual ou superior a 1/2 SM. A chance de estatura baixa também foi maior nas crianças que viviam em residências invadidas ou cedidas e nas crianças de domicílios com seis ou mais moradores. O uso de cigarro na gravidez, a idade da mãe inferior a 20 anos à época do nascimento da criança e a baixa estatura materna re- 
Tabela 1. Distribuição dos casos, controles, odds ratio (OR) e respectivo intervalo com 95\% de confiança (IC 95\%), segundo condições socioeconômicas, antecedentes maternos e características das crianças. João Pessoa, 2009.

\begin{tabular}{|c|c|c|c|c|c|}
\hline Variáveis & Total & Caso & Controle & OR (IC 95\%) & $\mathrm{p} \chi^{2}$ \\
\hline \multicolumn{6}{|l|}{ Nível distal: condições socioeconômicas } \\
\hline Renda familiar per capita* & & & & & 0,0003 \\
\hline$\geq 1 / 2 \mathrm{SM}$ & 40 & 13 & 27 & 1 & \\
\hline$<1 / 2 \mathrm{SM}$ & 94 & 54 & 40 & $2,80(1,6-4,9)$ & \\
\hline Tipo de casa & & & & & 0,0056 \\
\hline Própria/alugada & 33 & 12 & 21 & 1 & \\
\hline Invadida/cedida & 101 & 55 & 46 & $2,09(1,3-3,6)$ & \\
\hline Número de cômodos no domicílio & & & & & 0,0732 \\
\hline$\geq 4$ & 79 & 36 & 43 & 1 & \\
\hline$<4$ & 55 & 31 & 24 & $1,54(0,7-2,7)$ & \\
\hline Número de indivíduos no domicílio & & & & & 0,0061 \\
\hline$<6$ & 64 & 27 & 37 & 1 & \\
\hline$\geq 6$ & 70 & 40 & 30 & $1,83(1,1-3,2)$ & \\
\hline \multicolumn{6}{|l|}{ Nível intermediário: antecedentes maternos } \\
\hline Suplementação com sulfato ferroso na gravidez & & & & & 0,1901 \\
\hline Sim & 99 & 47 & 52 & 1 & \\
\hline Não & 35 & 20 & 15 & $1,47(0,4-3,1)$ & \\
\hline Número de consultas de pré-natal & & & & & 0,0533 \\
\hline$\geq 3$ & 109 & 53 & 56 & 1 & \\
\hline$<3$ & 25 & 14 & 11 & $1,34(0,8-2,7)$ & \\
\hline Uso de cigarro na gravidez & & & & & 0,0430 \\
\hline Não & 91 & 44 & 47 & 1 & \\
\hline Sim & 43 & 23 & 20 & $1,23(1,0-2,9)$ & \\
\hline Idade ao nascimento da criança (anos) & & & & & 0,0041 \\
\hline$\geq 20$ & 103 & 47 & 56 & 1 & \\
\hline$<20$ & 31 & 20 & 11 & $2,17(1,9-3,1)$ & \\
\hline Estatura para idade & & & & & 0,0001 \\
\hline Estatura normal & 97 & 43 & 54 & 1 & \\
\hline Baixa estatura $(<155,0 \mathrm{~cm})$ & 37 & 24 & 13 & $2,32(1,4-3,4)$ & \\
\hline \multicolumn{6}{|l|}{ Nível proximal: características das crianças } \\
\hline Sexo & & & & & 0,0841 \\
\hline Masculino & 71 & 34 & 37 & 1 & \\
\hline Feminino & 63 & 33 & 40 & $0,90(0,7-1,6)$ & \\
\hline Esquema vacinal ${ }^{*}$ & & & & & 0,0092 \\
\hline Completo & 92 & 45 & 47 & 1 & \\
\hline Incompleto & 33 & 19 & 14 & $1,42(1,3-3,2)$ & \\
\hline Peso ao nascer ${ }^{*}$ & & & & & 0,0039 \\
\hline Peso normal & 112 & 53 & 59 & 1 & \\
\hline Baixo peso ao nascer $(<2500 \mathrm{~g})$ & 13 & 8 & 5 & $1,78(1,2-2,9)$ & \\
\hline
\end{tabular}

SM: Salário Mínimo. ${ }^{* *}$ Considerando o valor do salário mínimo da época (R\$ 416,00). ${ }^{* *}$ Excluídas as crianças com informação ignorada (Caderneta de Saúde da Criança não apresentada).

presentaram chances de déficit de estatura nas crianças na ordem de 1,27; 2,17 e 2,32 vezes, respectivamente. O esquema vacinal incompleto da criança e o baixo peso ao nascer apresentaramse estatisticamente associados à estatura baixa.

Os resultados do modelo final da análise multivariada estão apresentados hierarquicamente na Tabela 2. Entre as variáveis socioeco- nômicas permaneceram como estatisticamente significativas a renda familiar per capita, o tipo de casa e o número de indivíduos no domicílio. A chance de a criança ter estatura baixa foi 2,93 vezes maior (IC 95\% = 1,5-5,7) nas famílias com renda familar per capita menor a 1/2 SM, em comparação àquelas que tinham renda superior a esse valor; 1,79 vezes maior (IC $95 \%=1,7-3,8$ ) 
Tabela 2. Odds Ratio (OR) e respectivo intervalo com 95\% de confiança (IC 95\%) dos fatores de risco resultantes da análise multivariada de casos e controles em relação ao déficit de estatura. João Pessoa, 2009.

\begin{tabular}{lcc}
\hline \multicolumn{1}{c}{ Variáveis } & OR & IC 95\% \\
\hline Nível distal: condições socioeconômicas & & \\
$\quad$ Renda familiar per capita $<1 / 2 \mathrm{SM}^{*}$ & 2,93 & $1,5-5,7$ \\
$\quad$ Casa invadida/cedida & 1,79 & $1,7-3,8$ \\
$\quad$ Número de indivíduos no domicílio $\geq 6$ & 1,85 & $1,4-4,0$ \\
Nível intermediário: antecedentes maternos & & \\
$\quad$ Idade ao nascimento da criança $<20$ anos & 1,68 & $1,1-3,4$ \\
$\quad$ Estatura para idade baixa $(<155,0 \mathrm{~cm})$ & 2,91 & $2,3-5,0$ \\
Nível proximal: características das crianças & & \\
$\quad$ Esquema vacinal incompleto & 1,26 & $1,3,9$ \\
Baixo peso ao nascer $(<2500 \mathrm{~g})$ & 2,69 & $2,4-2,8$ \\
\hline
\end{tabular}

SM: Salário Mínimo. ${ }^{*}$ Considerando o valor do salário mínimo da época (R\$ 416,00). * Ajustadas pelas outras variáveis do nível distal, incluídas no modelo. ${ }^{* *}$ Ajustadas pelas variáveis do nível distal e pelas outras variáveis do nível intermediário, incluídas no modelo. ${ }^{* * *}$ Ajustadas pelas variáveis dos níveis distal e intermediário e pelas outras variáveis do nível proximal, incluídas no modelo.

se a criança residia em casa invadida ou cedida, em comparação àquelas que residiam em casa própria ou alugada; e 1,85 vezes maior (IC 95\% $=1,4-4,0)$ se a criança morava em domicilio com seis ou mais moradores, em comparação àquelas que moravam em domicilio com menos de seis moradores. Das variáveis relacionadas aos antecedentes maternos, a idade inferior a 20 anos à época do nascimento da criança e a estatura para a idade permaneceram como importantes fatores preditores da baixa estatura da criança. Mantiveram-se também associadas à estatura baixa da criança, após ajuste com as variáveis dos blocos anteriores, o esquema vacinal da criança incompleto e o baixo peso ao nascer. Das variáveis materno-infantis, a baixa estatura para idade materna (odds ratio $=2,91$ e IC 95\% $=2,3$ $5,0)$ e o baixo peso ao nascer da criança (odds ratio $=2,69$ e IC $95 \%=2,4-2,8)$ foram as mais fortemente associadas.

\section{Discussão}

Apesar das possíveis semelhanças pelo contexto relacionado à institucionalização das crianças em creches, a caracterização da população de estudo indica exposições diferenciadas. Assim, é viável a identificação de fatores de risco por meio do presente estudo, com a utilização de um caso-controle dentro de uma creche.

A análise do modelo final destaca a determinação do déficit de estatura por variáveis dos três níveis (condições socioeconômicas, antecedentes maternos, características das crianças), representando a interação de múltiplos fatores na expressão do crescimento linear ${ }^{15}$. A compreensão simplificada desses fatores considerando a influência genética e as condições ambientais ${ }^{15,16}$ também está manifesta nos resultados: a estatura materna como fator de risco de ordem genético e as características socioeconômicas, por exemplo, de ordem ambiental. Nesse contexto, cabe ressaltar que, como os fatores genéticos praticamente não mudam de geração para geração ${ }^{15}$ e a estatura da mãe também pode resultar do efeito acumulado da pobreza em várias gerações em um processo cíclico que se inicia desde a vida intrauterina ${ }^{17}$, a variabilidade no crescimento linear condicionase basicamente às condições de vida.

O modelo hierárquico ${ }^{13}$ de determinação do déficit de estatura adotado neste trabalho postula a influência de fatores de diferentes níveis de complexidade. Nesse sentido, as condições socioeconômicas comportaram-se como importantes preditores do estado nutricional infantil, interferindo imediatamente nas características maternas (idade ao nascimento da criança, estatura para idade). Por sua vez, as características maternas repercutiriam nas condições das crianças, determinantes do último nível representados pela ocorrência de baixo peso ao nascer e incompletitude do esquema vacinal.

É reconhecido que o crescimento linear constitui um excelente indicador de desigualdades sociais ou do bem-estar populacional ${ }^{15,18}$. Estudos recentes sobre as causas do declínio do déficit de estatura no Brasil têm indicado, entre outros fa- 
tores, a importância da condição socioeconômica e do poder aquisitivo das famílias ${ }^{3,5,7,19}$. A maior vulnerabilidade das crianças de famílias pobres relacionada ao retardo do crescimento foi claramente estabelecida em ampla revisão da literatura que descreveu o efeito das iniquidades sociais sobre a nutrição e a saúde de crianças de países de renda baixa e média. No estudo, o maior risco de desnutrição nas crianças de famílias mais pobres explica-se pela maior suscetibilidade à exposição a agentes patogênicos que aumentam o risco de adoecer associado à menor resistência orgânica $\mathrm{e}$ à menor cobertura de ações preventivas; doentes, essas crianças também enfrentam as desigualdades relacionadas aos serviços de saúde ${ }^{20}$. Esta abordagem apresenta uma perspectiva que delineia a determinação social da saúde como princípio básico da prevenção do déficit de estatura, tal como relatam Bacallao et al. ${ }^{21}$.

A correlação positiva entre o estado nutricional materno e o dos filhos tem sido anteriormente argumentada pelo compartilhamento de informações genéticas e ambientais ${ }^{17,18}$. Nesse sentido, os estudos convergem na forte associação entre a estatura baixa de mães e crianças ${ }^{6,17,18,22-25}$. Sugere-se que a desnutrição crônica insere-se num processo cíclico de desnutrição infantil, déficit de estatura, obesidade e comorbidades na vida adulta ${ }^{18,23}$, no qual a baixa estatura materna como importante preditor da desnutrição em crianças associa-se à pobreza e às condições ambientais adversas ${ }^{23}$.

Apesar de a natureza multicausal do déficit de estatura em crianças considerar a importância da idade materna do ponto de vista reprodutivo $^{26}$, vários estudos ${ }^{6,15,26,27}$, com desenhos diferentes, divergem da associação encontrada neste trabalho. Entretanto, estudos anteriores também mostraram associação entre a idade materna e o crescimento linear das crianças ${ }^{23,28}$. Ainda que a idade materna apareça na literatura como variável de importância para o crescimento infantil, porém sem resultados que possibilitem conclusões, há que considerar que a mãe representa importante interface entre a criança e o ambiente $^{23}$, o que implica a necessidade de priorizar a vigilância nutricional das mães adolescentes em função do maior risco nutricional dos seus fi- $\operatorname{lhos}^{28}$. É importante notar que a variável analisada neste momento foi a idade da mãe ao nascimento da criança, especificidade não encontrada nos estudos revisados.

No nível proximal, a associação entre baixo peso ao nascer e o esquema vacinal incompleto com o déficit de estatura pode refletir a precariedade dos serviços de assistência à saúde. O peso ao nascer repercute na estatura infantil pela intermediação de fatores, como os cuidados durante a gestação e o estado nutricional materno durante a gravidez, que se expressam nos determinantes imediatos do crescimento linear ${ }^{29,30}$. A imunização, por sua importância no sistema imune e na resistência às infecções, repercute, também, na adequação da estatura em relação à idade da criança ${ }^{31}$. A associação controlada entre o baixo peso ao nascer e o déficit de estatura também foi encontrada em outros estudos ${ }^{23,28,30}$, bem como entre o esquema vacinal incompleto e o déficit de estatura ${ }^{18,32}$.

Os resultados do presente estudo, além de serem importantes devido ao efeito do crescimento linear na saúde e desenvolvimento infantil ${ }^{6-8}$, são essências para o campo da saúde coletiva, pois a redução da desnutrição infantil no Brasil representa um bom exemplo do impacto das políticas públicas no perfil epidemiológico, minimizando a dificuldade do conhecimento epidemiológico se constituir em prática transformadora das condições de saúde populacionais. Nesse sentido, o perfil epidemiológico do estado nutricional das crianças brasileiras tem sido favorecido por políticas públicas relacionadas a melhorias na escolaridade materna, no poder de compra das famílias, na assistência materno-infantil e na rede de água e esgoto ${ }^{33}$.

Em conclusão, fica evidente que, ao lado dos fatores socioeconômicos, aparecem, também, como fatores de risco da baixa estatura em crianças, características materno-infantis. A estatura materna sobressai por ser uma característica que expressa condição genética e ambiental. Por sua vez, a imunização e o peso ao nascer expressam as possíveis consequências positivas de serviços públicos de saúde de qualidade, principalmente em condições sociais, econômicas e ambientais desfavoráveis. 


\section{Colaboradores}

Figueroa Pedraza D participou da elaboração do protocolo de estudo, concepção, revisão bibliográfica, análise e interpretação dos dados, redação e revisão final do artigo. TN Menezes participou da análise e interpretação dos dados, redação e revisão final do artigo.

\section{Referências}

1. Onis Mercedes, Blossner M, Borghi E. Prevalence and trends of stunting among pre-school children, 1990-2020. Public Health Nutr 2012; 15(1):142-148.

2. Fundo das Nações Unidas para a Infância (UNICEF). Situação mundial da infância. New York: UNICEF; 2011.

3. Monteiro CA, Benicio MHD'A, Silva ACF, Lima ALL, Conde WL. Causas do declínio da desnutrição infantil no Brasil, 1996-2007. Rev Saude Publica 2009; 43(1):35-43.

4. Figueroa Pedraza D, Rocha ACD, Sousa CPC. Crescimento e deficiências de micronutrientes: perfil das crianças assistidas no Núcleo de Creches do Governo da Paraíba. Cien Saude Colet 2013; 18(11): 3379-3390.

5. Monteiro CA, Benicio MHD'A, Conde WL, Konno S, Lovadino AL, Barros AJD, Victoria CG. Narrowing socioeconomic inequality in child stunting: the Brazilian experience, 1974-2007. Bull World Health Organ 2010; 88(4):305-311.

6. Rehman AM, Gladstone BP, Verghese VP, Muliyil J, Jaffar S, Kang G. Chronic growth faltering amongst a birth cohort of Indian children begins prior to weaning and is highly prevalent at three years of age. Nutrition Journal 2009; 8(44):1-11.

7. Leal VS, Lira PIC, Menezes RCE, Oliveira JS, Sequeira LAS, Andrade SLLS, Batista Filho M. Fatores associados ao declínio do déficit estatural em crianças e adolescentes em Pernambuco. Rev Saude Publica 2012; 46(2):234-241.

8. Assis AMO, Barreto ML, Oliveira LPM, Oliveira VA, Prado MS, Gomes GSS, Pinheiro SMC, Santos NS, Silva RCR, Sampaio LR, Santos LMP. Determinants of mild-to-moderate malnutrition in preschoolers in an urban area of Northeastern Brazil: a hierarchical approach. Public Health Nutrition 2007; 11(4):387-394.

9. World Health Organization (WHO). Physical status: the use and interpretation of anthropometry. Geneva: WHO; 1995. (Technical Report Series 854).

10. World Health Organization (WHO). WHO Child Growth Standards. Length/height-for-age, weight-forage, weight-for-length, weight-for-height and body mass index-for-age. Methods and development. Geneva: WHO; 2006.

11. Onis M, Onyango AW, Borghi E, Siyam A, Nishida C, Siekmanna J. Development of a WHO growth reference for school-aged children and adolescents. Bull World Health Org 2007; 85(9):660-667.

12. National Center for Health Statistics. Centers for Disease Control and Prevention. National Health and Nutrition Examination Survey. USA: CDC; 2000. [Internet]. [acessado $2012 \mathrm{dez}$ 1]. Disponível em: http://www.cdc.gov/growthcharts

13. Victora CG, Huttly SR, Fuchs SC, Olinto MT. The role of conceptual frameworks in epidemiological analysis: a hierarquical approach. Int J Epidemiol 1997; 26(1):224-227.

14. Brasil. Ministério da Saúde (MS). Conselho Nacional de Saúde. Resolução no 196 de 10 de outubro de 1996. Diretrizes e Normas Regulamentadoras de Pesquisas Envolvendo Seres Humanos. Diário Oficial da União 1996; 16 out. 
15. Figueiroa JN, Alves JGB, Lira PIC, Batista Filho M. Evolução intergeracional da estatura no Estado de Pernambuco, Brasil, entre 1945 e 2006. 2 - aspectos analíticos. Cad Saude Publica 2012; 28(8):1468-1478.

16. Figueiroa JN, Alves JGB, Lira PIC, Batista Filho M. Evolução intergeracional da estatura no Estado de Pernambuco, Brasil, entre 1945 e 2006. 1 - aspectos descritivos. Cad Saude Publica 2012; 28(7):1285-1296.

17. Sousa CPC, Sousa MPC, Rocha ACD, Figueroa Pedraza D. Perfil epidemiológico do estado nutricional de crianças assistidas em creches no Estado da Paraíba. Nutrire 2011; 36(1):111-126.

18. Souza MM, Figueroa Pedraza D, Menezes TN. Estado nutricional de crianças assistidas em creches e situação de (in)segurança alimentar de suas famílias. Cien Saude Colet 2012; 17(12):3425-3436.

19. Monteiro CA, Benicio MHD’A, Silva ACF, Lima ALL, Conde WL. Causas do declínio acelerado da desnutrição infantil no Nordeste do Brasil (19861996-2006). Rev Saude Publica 2010; 44(1):17-27.

20. Barros FC, Victora CG, Scherphier R, Gwatkin D. Socioeconomic inequities in the health and nutrition of children in low/middle income countries. Rev Saude Publica 2010; 44(1):1-16.

21. Bacallao J, Peña M, Díaz A. Reducción de la desnutrición crónica en las bases biosociales para la promoción de la salud y el desarrollo. Rev $\mathrm{Pa}$ nam Salud Publica 2012; 32(2):145-150.

22. Marinho SP, Ignez S, Oliveira DC, Araújo EAC. Obesidade e baixa estatura: estado nutricional de indivíduos da mesma família. Rev Bras Crescimento Desenvolv Hum 2007; 17(1):156-164.

23. Silveira KBR, Alves JFR, Ferreira HS, Sawaya AL, Florêncio TMMT. Association between malnutrition in children living in favelas, maternal nutritional status and environmental factors. $J$ Pediatr 2010; 86(3):215-220.

24. Martins IS, Marinho SP, Oliveira DC, Araújo EAC. Pobreza, desnutrição e obesidade: inter-relação de estados nutricionais de indivíduos de uma mesma família. Cien Saude Colet 2007; 12(6):1553-1565.

25. Menezes RCE, Lira PIC, Leal VS, Oliveira JS, Santana SCS, Sequeira LAS, Rissin A, Batista Filho M. Determinantes do déficit estatural em menores de cinco anos no Estado de Pernambuco. Rev Saude Publica 2011; 45(6):1079-1087.
26. Rissin A, Figueiroa JN, Benicio MHD’A, Batista Filho M. Retardo estatural em menores de cinco anos: um estudo "baseline". Cien Saude Colet 2011; 16(10):4067-4076.

27. Zöllner CC, Fisberg RM. Estado nutricional e sua relação com fatores biológicos, sociais e demográficos de crianças assistidas por creches da Prefeitura do Município de São Paulo. Rev Bras Saúde Matern Infant 2006; 6(3):319-328.

28. Vitolo MR, Gama CM, Bortolini GA, Campagnolo PDB, Drachler ML. Some risk factors associated with overweight, stunting and wasting among children under 5 years old. J Pediatr 2008; 84(3):251-257.

29. Oliveira VA, Assis AMO, Pinheiro SMC, Barreto ML. Determinantes dos déficits ponderal e de crescimento linear de crianças menores de dois anos. Rev Saude Publica 2006; 40(5):874-882.

30. Orlonski S, Dellagrana RA, Rech CR, Araújo EDS. Estado nutricional e fatores associados ao déficit de estatura em crianças atendidas por uma unidade de ensino básico de tempo integral. Rev Bras Crescimento Desenvolv Hum 2009; 19(1):54-62.

31. Victoria CG, Aquino EML, Leal MC, Monteiro CA, Barros FC, Szwarcwald CL. Saúde de mães e crianças no Brasil: progressos e desafios. Lancet [periódico na Internet] $2011 \mathrm{Mai}$ [acessado $2013 \mathrm{fev}$ 10]:3246. Disponível em: http://download.thelancet.com/ flatcontentassets/pdfs/brazil/brazilpor2.pdf.

32. Oliveira LPM, Barreto ML, Assis AMO, Braga-Junior ACR, Nunes MFFP, Oliveira NF, Benício MHD'A, Venâncio SI, Saldiva SRDMk, Escuder MML. Preditores do retardo de crescimento linear em pré-escolares: uma abordagem multinível. Cad Saude Publica 2007; 23(3):601-613.

33. Barata RB. Epidemiologia e políticas públicas. Rev Bras Epidemiol 2013; 16(1):3-17.

Artigo apresentado em 31/10/2013

Aprovado em 24/11/2013

Versão final apresentada em 03/12/2013 